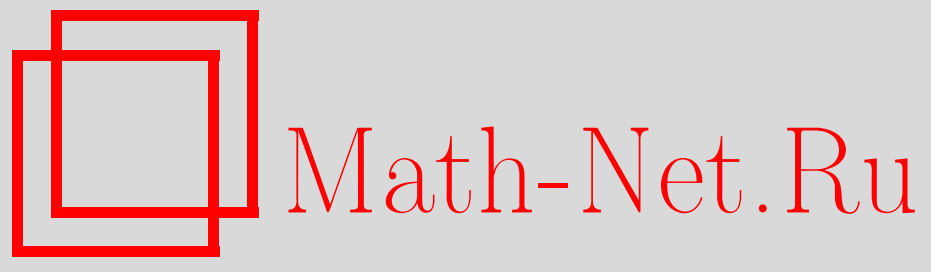

Д. Д. Киселев, Примеры задач погружения, у которых решения только поля, УМН, 2013, том 68, выпуск 4, 181182

DOI: https://doi.org/10.4213/rm9523

Использование Общероссийского математического портала Math-Net.Ru подразумевает, что вы прочитали и согласны с пользовательским соглашением http://www . mathnet.ru/rus/agreement

Параметры загрузки:

IP : 3.85 .73 .92

26 апреля 2023 г., $14: 10: 41$

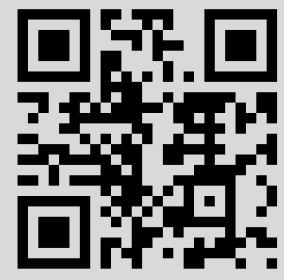




\section{Примеры задач погружения, у которых решения только поля}

\section{Д. Д. Киселев}

Задача погружения состоит в том, чтобы по данному расширению конечных групп

$$
1 \longrightarrow N \longrightarrow G \stackrel{\varphi}{\longrightarrow} \operatorname{Gal}(K / k) \longrightarrow 1
$$

построить алгебру Галуа $L$ над полем $k$, содержащую $K$ в качестве подполя и имеющую над $k$ группу автоморфизмов $G$, таким образом, чтобы эпиморфизм ограничения этих автоморфизмов на поле $K$ совпадал бы с $\varphi$. Хорошо известно (см. $[1 ;$ гл. $1, \S 6$, следствие 5]), что если ядро $N$ задачи погружения $(K / k, G, \varphi, N)$, порождаемой расширением (1), лежит в группе Фраттини $\Phi(G)$ группы $G$, то всякое решение такой задачи необходимо будет полем.

Целью настоящей заметки является построение серии примеров групповых расширений вида (1), для которых соответствующая задача погружения имеет только поля в качестве решений, но ядро при этом не лежит в группе Фраттини объемлющей группы. Заметим, что такая постановка задачи на момент написания статьи в литературе не рассматривалась.

Лемма 1 (Д.К. Фаддеев). Задача $\left(\mathbb{Q}(\sqrt{3}) / \mathbb{Q}, Q_{8}, \varphi,\langle j\rangle\right)$ разрешима, причем все решения такой задачи являются полями.

ДокАзАтельство. Из теории алгебр Галуа, изложенной, например, в $[1 ;$ гл. 1 , $\S 6]$, следует, что все решения разрешимой задачи погружения будут полями тогда и только тогда, когда все присоединенные к ней задачи (см. определение в [2]) неразрешимы.

Результат Д. К. Фаддеева (см. [3], [4]) гласит, что любая задача $\left(k(\sqrt{d}) / k, Q_{8}, \gamma, Z_{4}\right)$, где $2 \nmid \operatorname{char} k$, разрешима тогда и только тогда, когда $d$ представимо в виде суммы трех квадратов элементов поля $k$. Так как 3 представима в виде суммы трех квадратов элементов поля $\mathbb{Q}$, но не представима в виде суммы двух квадратов элементов поля $\mathbb{Q}$, то лемма полностью доказывается применением примера из $[1 ; \S 1]$, а также того факта, что любая присоединенная задача в данном случае имеет вид $\left(\mathbb{Q}(\sqrt{3}) / \mathbb{Q}, Z_{4}, \gamma, Z_{2}\right)$.

Возьмем произвольную задачу $(K / k, G, \varphi, N)$, все решения которой являются полями. Зафиксируем конечное расширение Галуа $L / k$ с группой $F_{1}$, содержащее $K$. Нас будет интересовать вопрос о том, когда все решения поднятой задачи $\left(L / k, G \times{ }_{F}\right.$ $\left.F_{1}, \bar{\varphi}, N\right)$ также являются полями. Здесь, конечно, $F=\operatorname{Gal}(K / k)$.

Лемма 2. Все решения поднятой задачи являются полями тогда и только тогда, когда произволъное решение $L_{1}$ исходной задачи линейно разделено с $L$ над $K$.

ДокАзАтЕльство. Из результатов параграфа 14 главы 1 книги [1] следует, что между решениями поднятой задачи и решениями исходной задачи имеется биективное соответствие: $L_{2} \leftrightarrow L_{2}^{N_{1}}$, где $N_{1}$ - ядро эпиморфизма ограничения $\theta: F_{1} \rightarrow F$. Отсюда непосредственно заключаем, что любое решение поднятой задачи имеет вид $A=$ $L_{1} \otimes_{K} L$, где $L_{1}-$ некоторое решение исходной задачи. Алгебра Галуа $A$ является полем тогда и только тогда, когда $L_{1} \cap L=K$. Лемма доказана.

Заметим, что в силу транзитивности подъема поднятую задачу можно получить из исходной шагами, на каждом из которых подъем осуществляется до поля, группа Галуа которого над предыдущим полем проста. Иными словами, со всяким расширением Галуа $L / k$, содержащим $K$ в качестве подполя, можно связать башню полей

$$
k \subset K_{0}:=K \subset K_{1} \subset \cdots \subset K_{s-1} \subset K_{s}:=L,
$$

причем $\operatorname{Gal}\left(K_{i} / K_{i-1}\right)$ проста для любого $i=\overline{1, s}$.

DOI: $10.4213 /$ rm9523 
Теорема 1. Рассмотрим разрешимую задачу $(K / k, G, \varphi, N)$, все решения которой являются полями. Поднимем ее до задачи $\left(L / k, G \times_{F} F_{1}, \bar{\varphi}, N\right)$, где $F=$ $\operatorname{Gal}(K / k), F_{1}=\operatorname{Gal}(L / k)$. В указанных ниже достаточных условиях все решения поднятой задачи будут полями: либо (1) композичионные факторы группы $\operatorname{Gal}(L / K)$ являются неабелевыми простыми группами, а все подгруппы группы $N$ нормальны в $N$; либо (2) группа $\operatorname{Gal}(L / K)$ разрешима, а $N$ совпадает со своим коммутантом.

ДоКАЗАТЕЛЬСТво. В силу индуктивных соображений на основе рассмотрения башни вида (2) достаточно ограничиться случаем, когда $\operatorname{Gal}(L / K)$ проста. В случае (1) имеем линейную разделенность произвольного решения $L_{1}$ исходной задачи с $L$ над $K$. Действительно, $L_{1} \cap L \subset L_{1}$, поэтому $L_{1} \cap L / K$ является расширением Галуа. Но группа $\operatorname{Gal}(L / K)$ проста и неабелева, а группа $N$ заведомо будет нильпотентной, поэтому $L_{1} \cap L \neq L$.

В случае (2) группа $\operatorname{Gal}(L / K)$ есть циклическая группа простого порядка. Из условия на группу $N$ следует, что у нее нет нормальных подгрупп простого индекса. Поэтому опять $L_{1} \cap L \neq L$, где $L_{1}$ - произвольное решение исходной задачи.

Лемма 3. Пусть в разрешимой задаче $(K / k, G, \varphi, N)$ ядро $N$ не лежит в $\Phi(G)$. Поднимем данную задачу до задачи $\left(L / k, G \times{ }_{F} F_{1}, \bar{\varphi}, N\right)$. Тогда $N \nless \Phi\left(G \times{ }_{F} F_{1}\right)$.

ДоказАтельство. Пусть $N \leqslant \Phi\left(G \times{ }_{F} F_{1}\right)$. Тогда, в частности, $N$ лежит в пересечении $M$ всех максимальных подгрупп группы $G \times{ }_{F} F_{1}$, содержащих ядро $N_{1}$ естественного эпиморфизма ограничения $\theta: F_{1} \rightarrow F$. Ясно, что образ $N$ при каноническом эпиморфизме $\pi: G \times{ }_{F} F_{1} \rightarrow\left(G \times{ }_{F} F_{1}\right) / N_{1}$ лежит в образе группы $M$. Но образ $M$ есть группа $\Phi\left(\left(G \times_{F} F_{1}\right) / N_{1}\right)$. Тем самым мы получаем, что $N \leqslant \Phi(G)$, ибо $N \cap N_{1}=\{1\}$ в группе $G \times{ }_{F} F_{1}$. Противоречие.

Теорема 2. Существует бесконечное семейство неабелевых простых групп $\mathfrak{G}$ такое, что для произволъной группы $N_{1}$, композичионные факторы которой принадлежат $\mathfrak{G}$, задача погружения для произвольного расширения

$$
1 \longrightarrow N_{1} \longrightarrow F_{1} \stackrel{\theta}{\longrightarrow} F=\operatorname{Gal}(\mathbb{Q}(\sqrt{3}) / \mathbb{Q}) \longrightarrow 1
$$

имеет поле-решение $L$. В частности, все решения поднятой задачи $\left(L / \mathbb{Q}, Q_{8} \times_{F} F_{1}\right.$, $\bar{\varphi}, N)$ являются полями, но $N \nless \Phi\left(Q_{8} \times_{F} F_{1}\right)$.

ДокАзАтельство. Согласно результатам книги [5] (см. гл. IV, § 3, следствие 3.7, а также гл. IV,$\S 4$, теорему 4.3 ) искомое поле $L$ действительно найдется, если в качестве $N_{1}$ взять произвольную группу, композиционные факторы которой перечислены в [5; гл. IV,$\S 4$, теорема 4.3]. Применяя леммы 1,3 и теорему 1 , получим нужный результат.

Автор благодарит Б. Б. Лурье за предложение рассмотреть поднятую задачу погружения. Автор благодарит также А.В. Яковлева и И. А. Чубарова за полезные обсуждения.

\section{Список литературы}

[1] В. В. Ишханов, Б. Б. Лурье, Д. К. Фаддеев, Задача погружения в теории Галуа, Наука, М., 1990, 270 с. [2] В. В. Ишханов, Б. Б. Лурье, Алгебра и анализ, 9:4 (1997), 87-97. [3] Д. К. Фаддеев, Уч. зап. Ленингр. гос. ун-та, 3:17 (1937), 17-23. [4] Д. К. Фаддеев, Докл. AH CCCP, 47:6 (1945), 404-407. [5] G. Malle, B. H. Matzat, Inverse Galois theory, Springer Monogr. Math., Springer-Verlag, Berlin, 1999, xvi+436 pp.

\section{Д. Д. Киселев (D. D. Kiselev)}

Московский государственный университет им. М. В. Ломоносова

E-mail: denmexmath@yandex.ru
Представлено Э.Б. Винбергом Принято редколлегией 05.04.2013 